ARTICLE

DOI: $10.1038 / \mathrm{s} 41467-018-05951-6$

\title{
Intermolecular selective carboacylation of alkenes via nickel-catalyzed reductive radical relay
}

Xian Zhao ${ }^{1}$, Hai-Yong Tu¹, Lei Guo ${ }^{1}$, Shengqing Zhu¹, Feng-Ling Qing ${ }^{1,2}$ \& Lingling Chu (1) ${ }^{1}$

The development of catalytic carboacylation of simple olefins, which would enable the rapid construction of ketones with high levels of complexity and diversity, is very challenging. To date, the vast majority of alkene carboacylation reactions are typically restricted to singleand two-component methodologies. Here we describe a three-component carboacylation of alkenes via the merger of radical chemistry with nickel catalysis. This reaction manifold utilizes a radical relay strategy involving radical addition to an alkene followed by alkyl radical capture by an acyl-nickel complex to forge two vicinal $\mathrm{C}-\mathrm{C}$ bonds under mild conditions. Excellent chemoselectivity and regioselectivity have been achieved by utilizing a pendant weakly chelating group. This versatile protocol allows for facile access to a wide range of important $\beta$-fluoroalkyl ketones from simple starting materials.

\footnotetext{
${ }^{1}$ State Key Laboratory for Modification of Chemical Fibers and Polymer Materials, College of Chemistry, Chemical Engineering and Biotechnology, Center for Advanced Low-Dimension Materials, Donghua University, Shanghai 201620, China. ${ }^{2}$ Key Laboratory of Organofluorine Chemistry, Shanghai Institute of Organic Chemistry, Chinese Academy of Science, Shanghai 200032, China. These authors contributed equally: Xian Zhao, Hai-Yong Tu. Correspondence and requests for materials should be addressed to L.C. (email: lingling.chu1@dhu.edu.cn)
} 
K etones are important structural motifs prevalent in pharmaceuticals, agrochemicals and natural products ${ }^{1,2}$, as well as versatile intermediates for numerous valuable transformations. Transition metal-catalyzed cross coupling with acyl electrophiles has emerged as an efficient platform for ketones synthesis ${ }^{3-6}$, providing an attractive alternative to classical nucleophilic carbonyl addition and Friedel-Crafts acylation? Particularly, catalytic carboacylation of alkenes in the presence of transition metal catalysts has attracted considerable attention in synthetic chemistry ${ }^{8-25}$, due to the fact that: (i) alkenes are abundant and ubiquitous building blocks in chemical and material industries; (ii) this protocol installs multiple $\mathrm{C}-\mathrm{C}$ bonds across an olefin in one step, allowing for rapid access to complex ketones from simple starting materials.

To date, the vast majority of alkene carboacylation reactions proceed via intramolecular insertion of an acyl-metal intermediate into an alkene. As a result, this reaction is typically restricted to single-component variants, with very few exceptions of two-component reactions ${ }^{12,18}$. The development of fully intermolecular, three-component carboacylation of simple olefins, which would enable the rapid construction of ketone products with high levels of complexity and diversity, is highly desired and remains elusive. One main challenge to this approach is the propensity for decarbonylation from an acylmetal species ${ }^{26-28}$, which must intercept the alkene. Another challenge associated with a three-component variant is achieving regioselectivity and chemoselectivity. A number of elegant methods have been reported to control regioselectivity in transition metal-catalyzed three-component dicarbofunctionalization of alkenes. For instance, previous studies have demonstrated that employment of activated substrates can facilitate regiocontrol ${ }^{29-40}$. More recently, utilization of directing groups to achieve highly regioselective dicarbofunctionalization of alkenes has also been reported ${ }^{41-49}$. Nevertheless, the capability to achieve selective alkene functionalization in the presence of multiple double bonds remains a longstanding challenge.

Over the last decade, nickel-catalyzed cross-coupling has emerged as a powerful tool to forge $\mathrm{C}-\mathrm{C}$ bonds in chemical synthesis ${ }^{50-52}$. Particularly, the merger of radical chemistry with nickel catalysis has enabled the invention of numerous useful transformations ${ }^{51,52}$. We recently questioned whether radical-based nickel catalysis might offer an alternative pathway to facilitate the three-component carboacylation of alkenes. Specifically, we envisioned that a radical relay strategy, involving radical addition to an alkene followed by alkyl radical capture by an acyl-nickel species ${ }^{53-55}$ would bypass the challenging acyl-metal/alkene capture required in a conventional two-electron mode. Furthermore, we anticipated that a pendant chelating group could facilitate the capture of the alkyl radical by the nickel species, while also controlling chemoselectivity and regioselectivity via coordination. This strategy described was also validated in seminal work by Nevado and coworkers in which an allylic acetate could serve to stabilize a putative alkylnickel species after radical recombination $^{44}$. Given the increasing importance of fluoroalkyl moieties in the areas of medicinal, agrochemical, and material chemistry ${ }^{56,57}$ as well as elegant progress in radical fluoroalkylation of alkenes ${ }^{58-62}$, we sought to explore the carboacylation of alkenes with fluoroalkyl precursors. Herein, we report selective, three-component carboacylation of alkenes with acyl chlorides via Ni-catalyzed radical relay (Fig. 1). This method takes advantage of $\mathrm{Ni}$-catalyzed reductive coupling of electrophiles ${ }^{3,63-66}$ under mild conditions, delivering valuable $\beta$-fluoroalkyl carbonyls that are not easily accessible by other methods 66,67 . a Intermolecular, selective carboacylation of olefins via $\mathrm{Ni}$-cat radical relay

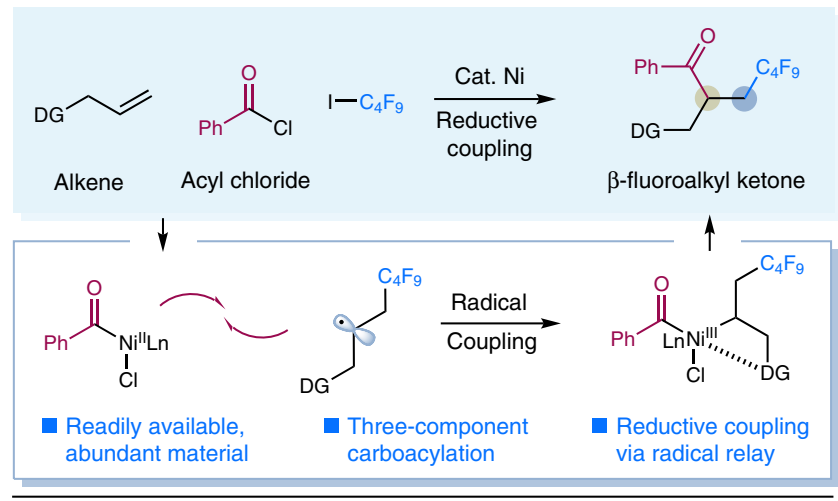

b Selectivity guided by a pendant chelating group

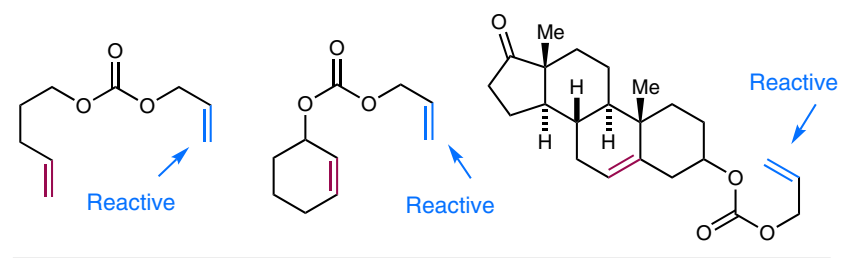

Fig. 1 Design of an intermolecular, selective carboacylation of alkenes. a Three-component carboacylation of olefins via nickel-catalyzed reductive radical relay. b Selectivity guided by a chelating group

\section{Results}

Optimization study. We first sought to employ carbonyls, ubiquitous intermediates in organic synthesis, as chelating groups 68 Evaluation of this nickel-catalyzed intermolecular reductive carboacylation strategy was first examined with allyl hexanoate, 4tert-butylbenzoyl chloride, and perfluorobutyl iodide in the presence of nickel catalysts and reductants. We were delighted to find that $93 \%$ yield of the desired carboacylation product could be obtained with $\mathrm{Mn}$ dust as stoichiometric reductant in the presence of catalytic amounts of $\mathrm{NiCl}_{2} \bullet$ glyme and $4,4^{\prime}$-di-tert-butyl$2,2^{\prime}$-dipyridyl (dtbbpy) at $25^{\circ} \mathrm{C}$ (See Supplementary Table 1). Control experiments have demonstrated that both the nickel catalyst and Mn dust are essential for the desired transformation to proceed, while moderate yield of $\mathbf{3}$ can be obtained in the absence of ligand, indicating the chelating ability of the pendant ester group (See Supplementary Table 1).

Substrate scope. With optimal reaction conditions in hand, we explored the generality of this carboacylation with respect to the alkene fragment and found that a variety of readily available alkenes are viable partners for this transformation (Fig. 2a). Alkenes tethered with different chelating groups, including esters, carbonates, carbamates, sulfonates, and phosphates, undergo the desired reductive coupling with moderate to excellent efficiency (products 1-9, 42-93\% yields). Interestingly, electron-rich aryl rings were capable of guiding the desired transformations ${ }^{69}$, exemplified by the reaction of aryl vinyl ethers to furnish $\alpha$-oxy$\beta$-fluoroalkyl ketones in high yields (products 10 and 11, 63\% and $76 \%$ yields, respectively). The mild reaction conditions allow for good compatibility with a wide range of important functional groups including aryl bromides and chlorides, providing a versatile platform for further synthetic manipulations (products 13, $74 \%$ yield). Gratifyingly, more complex partners derived from naturally occurring molecules were also successfully employed, demonstrating the potential applicability of this methodology in late-stage functionalization. For example, derivatives of borneol 


1.0 equiv. $\quad{ }_{1.0 \text { equiv. }}^{1-R_{\mathrm{f}}}$

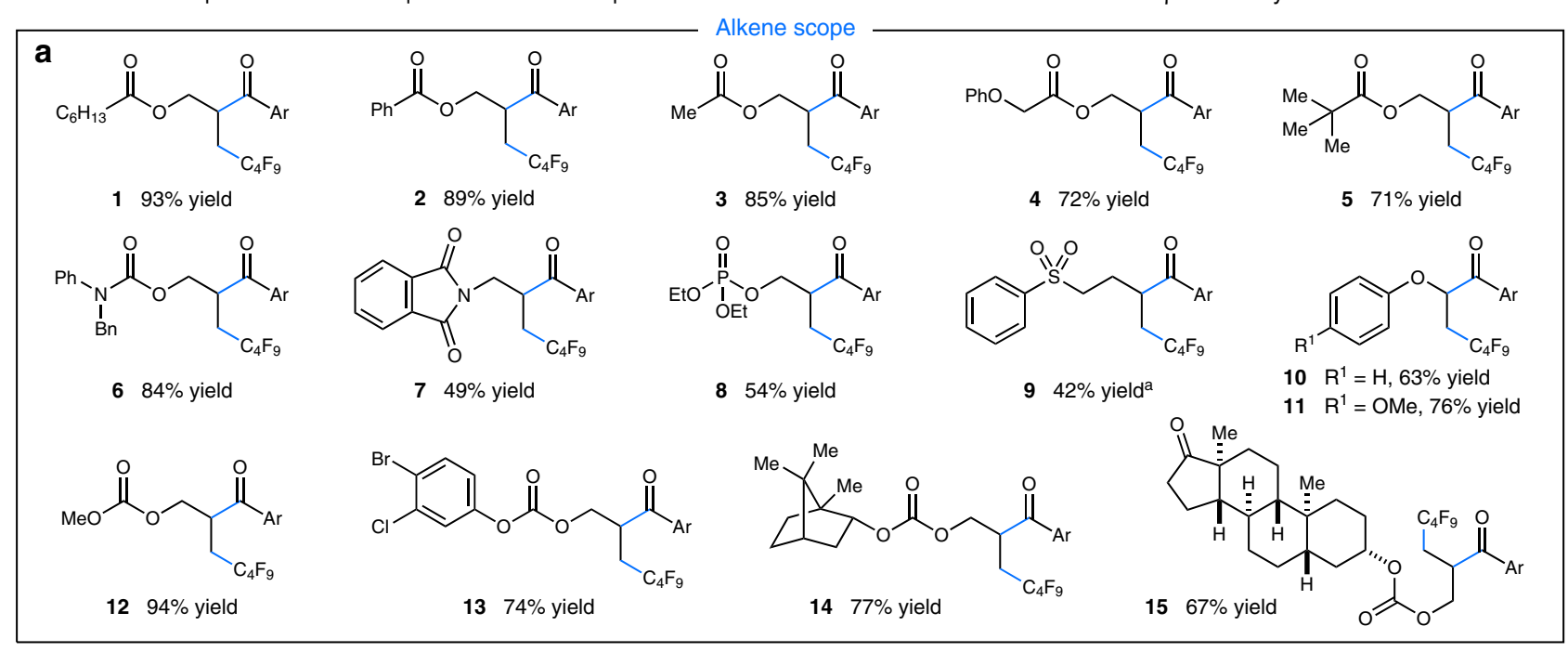

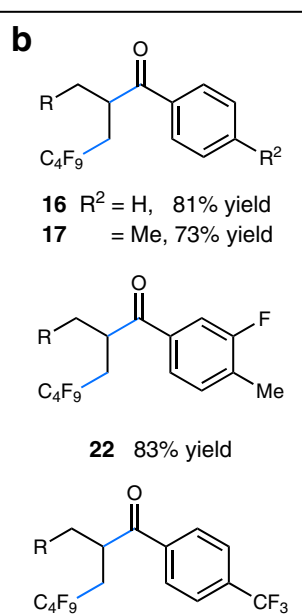

$2679 \%$ yield $^{a}$<smiles>O=C(c1ccc(P)cc1)C(CP)CC(F)(F)F</smiles>

$18 \mathrm{R}^{3}=\mathrm{F}, \quad 75 \%$ yield $19=\mathrm{CO}_{2} \mathrm{Me}, 49 \%$ yield ${ }^{\mathrm{b}}$<smiles>[R]CC(CC(F)(F)F)C(=O)c1cc([N+](=O)[O-])cc([N+](=O)[O-])c1</smiles>

$2374 \%$ yield<smiles>O=C(c1cccs1)C(CP)CC(F)(F)F</smiles>

$2772 \%$ yield<smiles>Cc1cc(C(=O)C(CP)CC(F)(F)F)ccc1F</smiles>

$2074 \%$ yield<smiles>COc1ccc(C(=O)C(CP)CC(F)(F)F)cc1</smiles>

$2461 \%$ yield<smiles>O=C(c1ccco1)C(CP)CC(F)(F)F</smiles>

$2858 \%$ yield $^{a}$<smiles>[2H]CC(CC(F)(F)F)C(=O)c1cccc(Cl)c1</smiles>

$2163 \%$ yield<smiles>[R]CC(CC(F)(F)F)C(=O)c1ccccc1[N+](=O)[O-]</smiles>

$2554 \%$ yield<smiles>[R]CC(CC(F)(F)F)C(=O)CCc1ccccc1</smiles>

$2942 \%$ yield

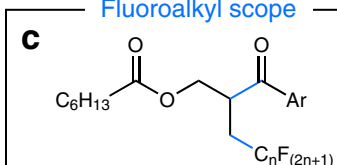

$30 \mathrm{C}_{3} \mathrm{~F}_{7}, 91 \% \quad 32 \mathrm{C}_{8} \mathrm{~F}_{17}, 78 \%$ $31 \mathrm{C}_{6} \mathrm{~F}_{13}, 85 \% 33 \mathrm{C}_{10} \mathrm{~F}_{21}, 71 \%^{\mathrm{c}}$<smiles>CCCCCCNC(=O)OCC(CC(=O)OCC)C(=O)[Al]</smiles>

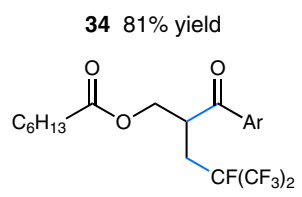

$3591 \%$ yield

Fig. 2 Substrate scope. a Scope of alkenes. b Scope of acyl chlorides. c Scope of fluoroalkyl iodides. Reaction conditions: $\mathrm{NiCl}_{2} \cdot \mathrm{glyme}(10 \mathrm{~mol} \%)$, dtbbpy (20 mol\%), alkene (1.0 equiv.), $\mathrm{R}_{\mathrm{f}} \mathrm{l}$ (1.0 equiv.), acyl chloride (1.5 equiv.), Mn (3.0 equiv.), $\mathrm{CH}_{3} \mathrm{CN}[0.1 \mathrm{M}], 25^{\circ} \mathrm{C}, 20 \mathrm{~h}$, see Supplementary Methods. All

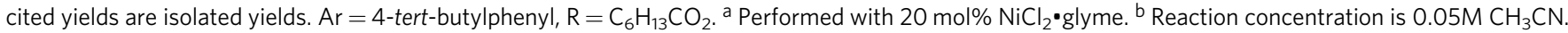
cPerformed in $\mathrm{CH}_{3} \mathrm{CN} / \mathrm{DME}$ (4:1) [0.1 M]

and estrone functioned as competent alkene partners, furnishing each of the desired coupling products with high efficiency (products 14 and $15,77 \%$ and $67 \%$ yields, respectively). Notably, acyclic internal alkenes, such as (E)-but-2-en-1-yl benzoate, also participated in this Ni-catalyzed difunctionalization manifold with moderate efficiency (see Supplementary Fig. 5).

Next, we evaluated the scope of the acyl chloride component in this protocol. As revealed in Fig. 2b, aromatic acyl chlorides containing electron-neutral, -donating, and -withdrawing groups proceeded smoothly under the optimal conditions, delivering the product ketones with moderate to excellent efficiency (products 16-26, $49-83 \%$ yields). Moreover, ortho substitution on the aryl ring was tolerated, albeit with somewhat diminished efficiency (product 25, 54\% yield). Notably, heteroaromatic acyl chlorides such as thiophene and furan were also viable substrates, furnishing the corresponding heteroaryl ketones in moderate yields (products 27 and 28, $72 \%$ and 58\% yields, respectively).
Additionally, aliphatic acyl chlorides demonstrated promising levels of efficiency, as exemplified by dialkyl ketone product $\mathbf{2 9}$ ( $42 \%$ yield).

Finally, we examined this three-component functionalization protocol with varied fluoroalkyl precursors (Fig. 2c). A series of perfluoroalkyl iodides, including ethyl iododifluoroacetate, can be readily employed with excellent levels of efficiency (products $\mathbf{3 0}$ $-35,53-91 \%$ yields), providing a simple and efficient way to incorporate perfluoroalkyl substituents into complex molecules. Importantly, this efficient alkene carboacylation strategy employs a 1:1 ratio of alkene and fluoroalkyl iodide at room temperature, without the need for excess amounts of perfluoroalkyl iodides in all cases (Fig. 2). Gratifyingly, trifluoroiodomethane $\left(\mathrm{CF}_{3} \mathrm{I}\right)$ was also reactive, affording the desired trifluoromethylacylation product in promising levels of efficiency (29\% yield) (see Supplementary Fig. 6). Additionally, electron-deficient tertiary alkyl bromides were also suitable coupling partners under slightly 
a Reactivity tuned by the chelating groups

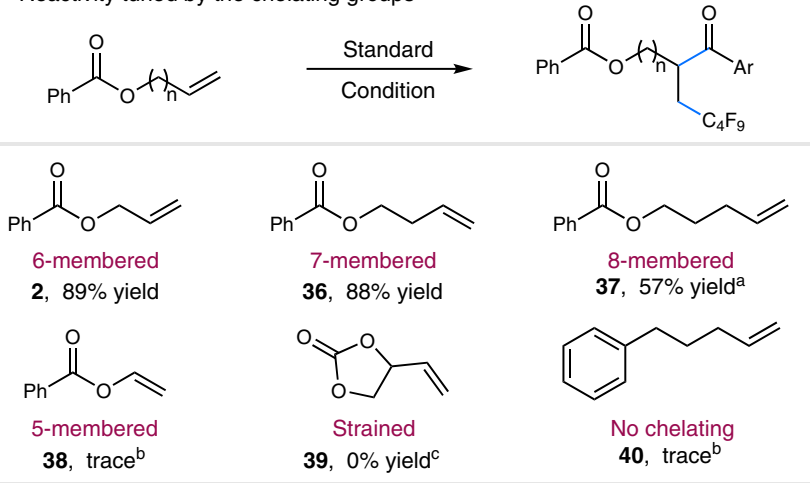

b Chemoselectivity in the presence multiple double bonds<smiles>C=CCCCOC(=O)OCC(CC(F)(F)F)C(=O)Br</smiles>

$4143 \%$ yield ${ }^{d, e}$,

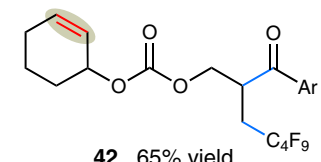

$4265 \%$ yield<smiles>CC(COC(=O)OCC(CC(F)(F)F)C(N)=O)[C@H]1CCC2=CCCC([N+](=O)[O-])C2(C)C1</smiles><smiles>O=C(OCC(CC(F)(F)F)C(=O)Br)Oc1ccc2oc(=O)ccc2c1</smiles>

$4468 \%$ yield

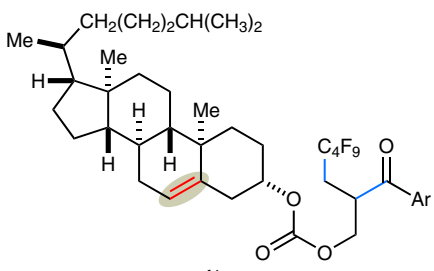

$4563 \%$ yield $^{\text {th }}$ (76\% brsm)

$4652 \%$ yield $^{\mathrm{f}, \mathrm{f}}$

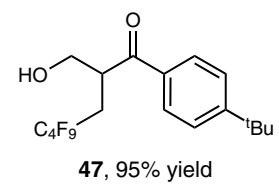<smiles>O=C(c1ccc(Br)cc1)C(C[18O])CN[18O]</smiles>

$49,70 \%$ yield<smiles>CC(C)C(CC(O)c1ccc(Br)cc1)CC(C)(F)F</smiles><smiles>CCCCC(CC(CC(C)(C)C)C(F)(F)F)C(=O)c1ccc(Br)cc1</smiles>

50, $56 \%$ yield

Fig. 4 Derivations of compound 3. See Supplementary Methods. Ar= 4-tert-butylphenyl. PMB $=4$-methoxybenzyl

bonds. As shown in Fig. 3b, our reductive protocol can be used for selective functionalization of alkenes through the directing effect. Exclusive site selectivity was observed for carboacylation of terminal alkenes that could form six-membered chelate rings, leaving the non-chelating alkenes untouched (products 41-46, $43-68 \%$ yields). Notably, for substrates in which two terminal alkenes are present, excellent site selectivity was observed at olefins guided by the tethered chelating group with a more favorable chelate geometry (products 41, 43\% yield), further highlighting the powerful selectivity feature of this catalytic system. Additionally, olefins derived from naturally occurring molecules, including valencene, coumarin, dehydroisoandrosterone, and cholesterone, could also be employed to furnish the desired products with moderate efficiency and excellent selectivity (products 43-46, 47-68\% yields), demonstrating the inherent value of our carboacylation protocol in late-stage functionalization.

To further demonstrate the synthetic benefit of our nickelcatalyzed reductive carboacylation strategy, the difunctionalized products were converted to several useful synthetic functionalities (Fig. 4). The acetyl group of compound 3 can be readily removed under acidic conditions to give alcohol 47 in $95 \%$ yield. Selective reduction of aryl ketones with $\mathrm{NaBH}_{4}$ led to the formation of secondary alcohol 48 in $80 \%$ yield. Furthermore, nucleophilic substitution of 3 with benzylamine afforded $\gamma$-fluoroalkylated amine 49 in $70 \%$ yield. $\gamma$-Fluoroalkylated thioether $\mathbf{5 0}$ was obtained in $56 \%$ yields through a $\beta$-elimination/Michael addition reaction with 4-methoxy- $\alpha$-toluenethiol.

Proposed mechanism and mechanistic studies. Our proposed mechanism for this Ni-catalyzed reductive coupling is outlined in Fig. 5a. Oxidative addition of the active $\mathrm{Ni}(0)$ species I to acyl chloride affords $\mathrm{Ni}^{\mathrm{II}}$ complex $\mathbf{I I}^{70}$. Concurrently, $\mathrm{Ni}(0)$ - or $\mathrm{Ni}^{\mathrm{I}}$ mediated single-electron reduction of $\mathrm{C}_{4} \mathrm{~F}_{9} \mathrm{I}$ generates the electrophilic $\mathrm{C}_{4} \mathrm{~F}_{9}$ radical, and subsequent radical addition to the alkene coupling partner would deliver alkyl radical species III. At this juncture, we expected that $\mathrm{Ni}^{\mathrm{II}}$ complex II could be intercepted by nucleophilic alkyl radical III to yield $\mathrm{Ni}^{\mathrm{III}}$ adduct IV $^{51,52}$, which would undergo reductive elimination to deliver the final product and $\mathrm{Ni}^{\mathrm{I}}$ species $\mathrm{V}$. Single-electron reduction of $\mathrm{Ni}^{\mathrm{I}}$ $\mathbf{V}\left(E_{\text {red }}\left[\mathrm{Ni}^{\mathrm{II}} / \mathrm{Ni}^{0}\right]=-1.2 \mathrm{~V} \text { vs SCE in } \mathrm{DMF}\right)^{71}$ by Mn dust $\left(E_{\text {red }}\right.$ $=-1.4 \mathrm{~V}$ vs SCE in $\mathrm{MeCN}$ ) would regenerate $\mathrm{Ni}(0)$ species I and complete the catalytic cycle. We expected that the chelating group would affect the feasibility and stability of the $\mathrm{Ni}^{\mathrm{III}}$ complex, thus influencing the reactivity of this reaction. Alternatively, alkyl radical III could be captured by $\mathrm{Ni}(0)$ to form $\mathrm{Ni}^{\mathrm{I}}$ complex $\mathrm{VII}^{72}$, followed by oxidative addition of acyl chloride to deliver the crucial $\mathrm{Ni}^{\mathrm{III}}$ adduct IV.

We have conducted several preliminary mechanistic experiments to elucidate the proposed mechanism (Fig. 5b-d). Addition 


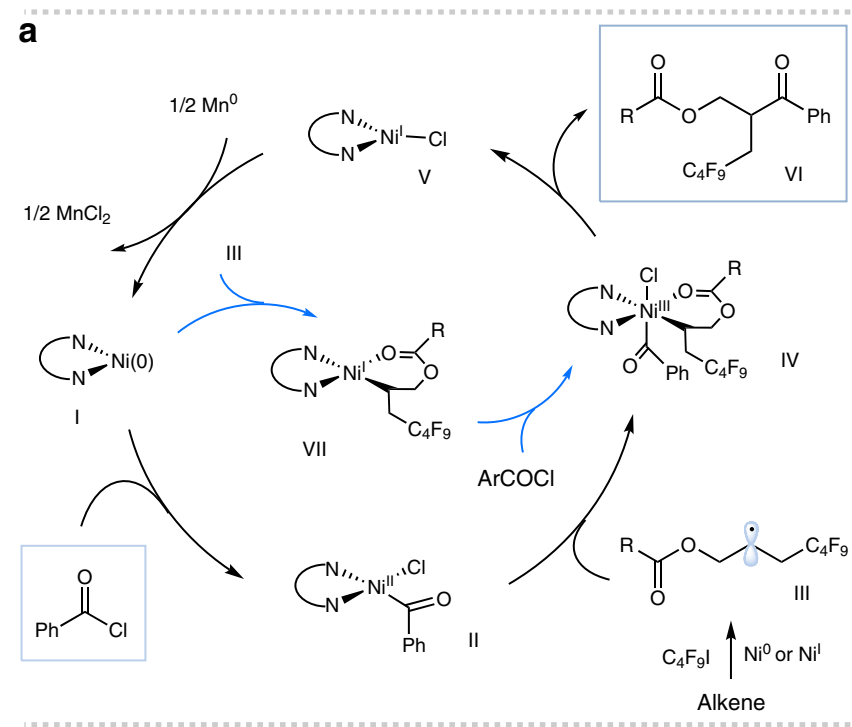

b

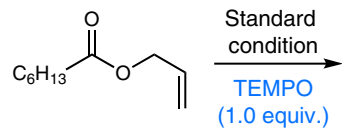<smiles>CCCCOC(=O)CC(COC(=O)C(F)(F)F)C(N)=O</smiles><smiles>CC1(C)CCCC(C)(C)N1OC(F)(F)C(F)(F)F</smiles>

$0.2 \mathrm{mmol}$

1, not observed

$\mathbf{5 1}, 79 \%$ yield

C<smiles>C=CCC(CC=C)(OCC)OCC</smiles>

52, $0.2 \mathrm{mmol}$

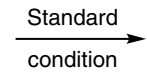<smiles>CCOC(=O)C1(OCC)CC(CC(=O)O)C(CC(F)(F)F)C1</smiles>

$53,23 \%$ yield

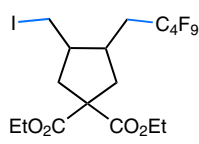

54, $49 \%$ yield d

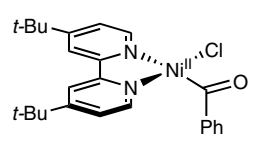

$55,0.1 \mathrm{mmol}$

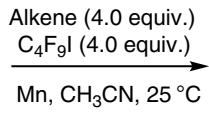<smiles>O=C(OCC(CC(F)(F)F)C(=O)c1ccccc1)c1ccccc1</smiles>

$16,42 \%$ yield
Fig. 5 Mechanistic studies. a Proposed mechanism. b Radical inhibition experiment. c Radical clock experiment. d Stoichiometric reaction of isolated $\mathrm{Ni}(\mathrm{II})$ complex. See Supplementary Discussion. $\mathrm{Ar}=4$-tertbutylphenyl. alkene = allyl heptanoate

of TEMPO (1.0 equiv.) completely shuts down the desired transformation, and TEMPO- $\mathrm{C}_{4} \mathrm{~F}_{9}$ adduct 51 was observed in $79 \%{ }^{19} \mathrm{~F}$ NMR yield (Fig. 5b). Moreover, diene 52 underwent radical addition and cyclization, furnishing the expected coupling product $\mathbf{5 3}$ in $23 \%$ yield as well as alkyl iodide $\mathbf{5 4}$ in $49 \%$ yield (Fig. 5c). These results indicate that radical intermediates are involved in this system. Importantly, the stoichiometric reaction of Ni-complex 55 with alkene and $\mathrm{C}_{4} \mathrm{~F}_{9} \mathrm{I}$ in the presence of $\mathrm{Mn}$ dust gave the desired coupling product 16 in $42 \%$ yield (Fig. 5 d), suggesting that the catalytic pathway proceeding via oxidative addition of $\mathrm{Ni}(0)$ species with acyl chloride could be operative (Fig. 5a).

\section{Discussion}

In conclusion, we have developed a robust strategy for intermolecular, three-component carboacylation of alkenes with acyl chlorides and fluoroalkyl iodides via a Ni-catalyzed radical relay. This versatile protocol enables facile access to $\beta$-fluoroalkyl ketones through the regioselective, sequential formation of two $\mathrm{C}$ $-\mathrm{C}$ bonds in one step under mild conditions. We expect that the generality of this methodology and readily availability of the starting materials will allow it to enjoy extensive application in the area of organic chemistry.

\section{Methods}

General procedure for the carboacylation reaction. To a flame-dried $8 \mathrm{~mL}$ reaction vial was charged with $\mathrm{NiCl}_{2} \bullet$ glyme $(0.02 \mathrm{mmol}, 10 \mathrm{~mol} \%), 4,4^{\prime}$-di-tert butyl-2,2'-dipyridyl ( $0.04 \mathrm{mmol}, 20 \mathrm{~mol} \%)$, and $\mathrm{Mn}$ ( $0.6 \mathrm{mmol}, 3.0$ equiv.). The vial was capped. After it was evacuated and backfilled nitrogen three times, $\mathrm{CH}_{3} \mathrm{CN}$ $[0.1 \mathrm{M}]$ was added via a syringe, followed by the addition of acyl chloride $(0.3 \mathrm{mmol}, 1.5$ equiv.). The reaction mixture was allowed to stir for approximately $1 \mathrm{~min}$ before fluoroalkyl iodide ( $0.2 \mathrm{mmol}, 1.0$ equiv., if liquid) and alkene $(0.2$ mmol, 1.0 equiv. if liquid) were added. The reaction mixture was allowed to stir at $1500 \mathrm{rpm}$ for $20 \mathrm{~h}$ at $25^{\circ} \mathrm{C}$. The reaction was quenched with $1 \mathrm{~N} \mathrm{HCl}$, extracted with ethyl acetate (EA) three times. The combined organic layers were dried with $\mathrm{MgSO}_{4}$, filtered, and concentrated in vacuo. The crude material was purified by flash chromatography to afford the product. See Supplementary Methods for further experimental details.

Data availability. The authors declare that all the data supporting the findings of this work are available within the article and its Supplementary Information files or from the corresponding author upon request.

Received: 5 April 2018 Accepted: 31 July 2018 Published online: 28 August 2018

\section{References}

1. Walter, M. W. Structure-based design of agrochemicals. Nat. Prod. Rep. 19, 278-291 (2002).

2. McDaniel, R. et al. Multiple genetic modifications of the erythromycin polyketide synthase to produce a library of novel "unnatural" natural products. Proc. Natl Acad. Sci. USA 96, 1846-1851 (1999).

3. Moragas, T., Correa, A. \& Martin, R. Metal-catalyzed reductive coupling reactions of organic halides with carbonyl-type compounds. Chem. Eur. J. 20, 8242-8258 (2014).

4. Willis, M. C. Transition metal catalyzed alkene and alkyne hydroacylation. Chem. Rev. 110, 725-748 (2010).

5. Gooßen, L. J., Rodríguez, N. \& Gooßen, K. Carboxylic acids as substrates in homogeneous catalysis. Angew. Chem. Int. Ed. 47, 3100-3120 (2008).

6. Dieter, R. K. Reaction of acyl chlorides with organometallic reagents: a banquet table of metals for ketone synthesis. Tetrahedron 55, 4177-4236 (1999).

7. Sartori, G. \& Maggi, R. Use of solid catalysts in Friedel-Crafts acylation reactions. Chem. Rev. 106, 1077-1104 (2006).

8. Chen, P.-h., Billett, B. A., Tsukamoto, T. \& Dong, G. "Cut and Sew" transformations via transition-metal-catalyzed carbon-carbon bond activation. ACS Catal. 7, 1340-1360 (2017).

9. Meng, G., Shi, S. \& Szostak, M. Cross-coupling of amides by N-C bond activation. Synlett 27, 2530-2540 (2016)

10. Souillart, L. \& Cramer, N. Enantioselective rhodium-catalyzed C-C bond activation of cyclobutanones. CHIMIA 69, 187-190 (2015).

11. Rong, Z. Q., Lim, H. N. \& Dong, G. Intramolecular acetyl transfer to olefins by catalytic C-C bond activation of unstrained ketones. Angew. Chem. Int. Ed. 57, 475-479 (2018)

12. Walker, J. A., Vickerman, K. L., Humke, J. N. \& Stanley, L. M. Ni-catalyzed alkene carboacylation via amide $\mathrm{C}-\mathrm{N}$ bond activation. J. Am. Chem. Soc. 139 10228-10231 (2017).

13. Lutz, J. P. et al. Rate-limiting step of the rh-catalyzed carboacylation of alkenes: C-C bond activation or migratory insertion? J. Am. Chem. Soc. 134, 715-722 (2012).

14. Xu, T., Ko, H. M., Savage, N. A. \& Dong, G. Highly enantioselective Rhcatalyzed carboacylation of olefins: efficient syntheses of chiral poly-fused rings. J. Am. Chem. Soc. 134, 20005-20008 (2012).

15. Liu, L., Ishida, N. \& Murakami, M. Atom- and step-economical pathway to chiral benzobicyclo[2.2.2] octenones through carbon-carbon bond cleavage. Angew. Chem. Int. Ed. 51, 2485-2488 (2012).

16. Rathbun, C. M. \& Johnson, J. B. Rhodium-catalyzed acylation with quinolinyl ketones: carbon-carbon single bond activation as the turnover-limiting step of catalysis. J. Am. Chem. Soc. 133, 2031-2033 (2011).

17. Dreis, A. M. \& Douglas, C. J. Catalytic carbon-carbon $\sigma$ bond activation: an intramolecular carbo-acylation reaction with acylquinolines. J. Am. Chem. Soc. 131, 412-413 (2009).

18. Wentzel, M. T., Reddy, V. J., Hyster, T. K. \& Douglas, C. J. Chemoselectivity in catalytic C-C and C-H bond activation: controlling intermolecular carboacylation and hydroarylation of alkenes. Angew. Chem. Int. Ed. 48, 6121-6123 (2009). 
19. Yatham Veera, R., Shen, Y. \& Martin, R. Catalytic intermolecular dicarbofunctionalization of styrenes with $\mathrm{CO}_{2}$ and radical precursors. Angew. Chem. Int. Ed. 56, 10915-10919 (2017).

20. McMahon, C. M., Renn, M. S. \& Alexanian, E. J. Manganese-catalyzed carboacylations of alkenes with alkyl iodides. Org. Lett. 18, 4148-4150 (2016).

21. Park, J.-W., Kou, K. G. M., Kim, D. K. \& Dong, V. M. Rh-catalyzed desymmetrization of [small alpha]-quaternary centers by isomerizationhydroacylation. Chem. Sci. 6, 4479-4483 (2015).

22. Ouyang, X.-H., Song, R.-J. \& Li, J.-H. Iron-catalyzed oxidative 1,2carboacylation of activated alkenes with alcohols: a tandem route to 3-(2oxoethyl)indolin-2-ones. Eur. J. Org. Chem. 2014, 3395-3401 (2014).

23. Seashore-Ludlow, B., Danielsson, J. \& Somfai, P. Domino carbopalladationcarbonylation: investigation of substrate scope. Adv. Synth. Catal. 354, 205-216 (2012).

24. Fusano, A., Sumino, S., Fukuyama, T. \& Ryu, I. Vicinal C-functionalization of alkenes. Pd/light-induced multicomponent coupling reactions leading to functionalized esters and lactones. Org. Lett. 13, 2114-2117 (2011).

25. Liu, C. \& Widenhoefer, R. A. Palladium-catalyzed cyclization/ carboalkoxylation of alkenyl indoles. J. Am. Chem. Soc. 126, 10250-10251 (2004).

26. Hu, J., Zhao, Y., Liu, J., Zhang, Y. \& Shi, Z. Nickel-catalyzed decarbonylative borylation of amides: evidence for Acyl $\mathrm{C}-\mathrm{N}$ bond activation. Angew. Chem. Int. Ed. 55, 8718-8722 (2016).

27. Shi, S., Meng, G. \& Szostak, M. Synthesis of Biaryls through nickel-catalyzed Suzuki-Miyaura coupling of amides by carbon-nitrogen bond cleavage. Angew. Chem. Int. Ed. 55, 6959-6963 (2016)

28. Johnson, J. B. \& Rovis, T. Enantioselective cross-coupling of anhydrides with organozinc reagents: the controlled formation of carbon-carbon bonds through the nucleophilic interception of metalacycles. Acc. Chem. Res. 41, 327-338 (2008).

29. Wu, L. et al. Asymmetric Cu-catalyzed intermolecular trifluoromethylarylation of styrenes: enantioselective arylation of benzylic radicals. J. Am. Chem. Soc. 139, 2904-2907 (2017).

30. Wang, F. et al. Enantioselective copper-catalyzed intermolecular cyanotrifluoromethylation of alkenes via radical process. J. Am. Chem. Soc. 138, 15547-15550 (2016).

31. Ouyang, X.-H., Song, R.-J., Hu, M., Yang, Y. \& Li, J.-H. Silver-mediated intermolecular 1,2-alkylarylation of styrenes with a-carbonyl alkyl bromides and indoles. Angew. Chem. Int. Ed. 55, 3187-3191 (2016).

32. $\mathrm{Wu}, \mathrm{X}$. et al. Enantioselective 1,2-difunctionalization of dienes enabled by chiral palladium complex-catalyzed cascade arylation/allylic alkylation reaction. J. Am. Chem. Soc. 137, 13476-13479 (2015).

33. Stokes, B. J., Liao, L., de Andrade, A. M., Wang, Q. \& Sigman, M. S. A palladium-catalyzed three-component-coupling strategy for the differential vicinal diarylation of terminal 1,3-dienes. Org. Lett. 16, 4666-4669 (2014).

34. Wang, F., Wang, D., Mu, X., Chen, P. \& Liu, G. Copper-catalyzed intermolecular trifluoromethylarylation of alkenes: mutual activation of arylboronic acid and $\mathrm{CF}_{3}{ }^{+}$reagent. J. Am. Chem. Soc. 136, 10202-10205 (2014).

35. McCammant, M. S., Liao, L. \& Sigman, M. S. Palladium-catalyzed 1,4difunctionalization of butadiene to form skipped polyenes. J. Am. Chem. Soc. 135, 4167-4170 (2013).

36. Liao, L., Jana, R., Urkalan, K. B. \& Sigman, M. S. A palladium-catalyzed threecomponent cross-coupling of conjugated dienes or terminal alkenes with vinyl triflates and boronic acids. J. Am. Chem. Soc. 133, 5784-5787 (2011).

37. Terao, J., Bando, F. \& Kambe, N. Ni-catalyzed regioselective three-component coupling of alkyl halides, arylalkynes, or enynes with $\mathrm{R}-\mathrm{M}(\mathrm{M}=\mathrm{MgX}, \mathrm{ZnX})$. Chem. Commun. 7336-7338 (2009)

38. Urkalan, K. B. \& Sigman, M. S. Palladium-catalyzed oxidative intermolecular difunctionalization of terminal alkenes with organostannanes and molecular oxygen. Angew. Chem. Int. Ed. 48, 3146-3149 (2009).

39. Shu, W., Jia, G. \& Ma, S. Palladium-catalyzed three-component cascade cyclization reaction of bisallenes with propargylic carbonates and organoboronic acids: efficient construction of cis-fused bicyclo[4.3.0]nonenes. Angew. Chem. Int. Ed. 48, 2788-2791 (2009).

40. Huang, T.-H., Chang, H.-M., Wu, M.-Y. \& Cheng, C.-H. Palladium-catalyzed three-component assembling of allenes, organic halides, and arylboronic acids. J. Org. Chem. 67, 99-105 (2002).

41. Thapa, S. et al. Ni-catalysed regioselective 1,2-diarylation of unactivated olefins by stabilizing Heck intermediates as pyridylsilyl-coordinated transient metallacycles. Chem. Sci. 9, 904-909 (2018).

42. Li, W., Boon, J. K. \& Zhao, Y. Nickel-catalyzed difunctionalization of allyl moieties using organoboronic acids and halides with divergent regioselectivities. Chem. Sci. 9, 600-607 (2018).
43. Derosa, J., Tran, V. T., Boulous, M. N., Chen, J. S. \& Engle, K. M. Nickelcatalyzed $\beta$, $\gamma$-dicarbofunctionalization of alkenyl carbonyl compounds via conjunctive cross-coupling. J. Am. Chem. Soc. 139, 10657-10660 (2017).

44. García-Domínguez, A., Li, Z. \& Nevado, C. Nickel-catalyzed reductive dicarbofunctionalization of alkenes. J. Am. Chem. Soc. 139, 6835-6838 (2017)

45. Shrestha, B. et al. Ni-catalyzed regioselective 1,2-dicarbofunctionalization of olefins by intercepting heck intermediates as imine-stabilized transient metallacycles. J. Am. Chem. Soc. 139, 10653-10656 (2017).

46. Zhang, L. et al. Catalytic conjunctive cross-coupling enabled by metal-induced metallate rearrangement. Science 351, 70-74 (2016).

47. Liu, Z., Zeng, T., Yang, K. S. \& Engle, K. M. $\beta$, $\gamma$-vicinal dicarbofunctionalization of alkenyl carbonyl compounds via directed nucleopalladation. J. Am. Chem. Soc. 138, 15122-15125 (2016).

48. Qin, T. et al. A general alkyl-alkyl cross-coupling enabled by redox-active esters and alkylzinc reagents. Science 352, 801-805 (2016).

49. Gu, J.-W., Min, Q.-Q., Yu, L.-C. \& Zhang, X. Tandem difluoroalkylationarylation of enamides catalyzed by nickel. Angew. Chem. Int. Ed. 55, 12270-12274 (2016).

50. Tasker, S. Z., Standley, E. A. \& Jamison, T. F. Recent advances in homogeneous nickel catalysis. Nature 509, 299-309 (2014).

51. Fu, G. C. Transition-metal catalysis of nucleophilic substitution reactions: a radical alternative to $\mathrm{SN}_{1}$ and $\mathrm{SN}_{2}$ processes. ACS Cent. Sci. 3, 692-700 (2017).

52. Twilton, J. et al. The merger of transition metal and photocatalysis. Nat. Rev. Chem. 1, 0052 (2017).

53. Zhang, X. \& MacMillan, D. W. C. Direct aldehyde C-H arylation and alkylation via the combination of nickel, hydrogen atom transfer, and photoredox catalysis. J. Am. Chem. Soc. 139, 11353-11356 (2017).

54. Amani, J. \& Molander, G. A. Synergistic photoredox/nickel coupling of acyl chlorides with secondary alkyltrifluoroborates: dialkyl ketone synthesis. J. Org Chem. 82, 1856-1863 (2017).

55. Joe, C. L. \& Doyle, A. G. Direct acylation of $\mathrm{C}\left(\mathrm{sp}^{3}\right)-\mathrm{H}$ bonds enabled by nickel and photoredox catalysis. Angew. Chem. Int. Ed. 55, 4040-4043 (2016)

56. Purser, S., Moore, P. R., Swallow, S. \& Gouverneur, V. Fluorine in medicinal chemistry. Chem. Soc. Rev. 37, 320-330 (2008).

57. Müller, K., Faeh, C. \& Diederich, F. Fluorine in pharmaceuticals: looking beyond intuition. Science 317, 1881-1886 (2007).

58. Egami, H. \& Sodeoka, M. Trifluoromethylation of alkenes with concomitant introduction of additional functional groups. Angew. Chem. Int. Ed. 53, 8294-8308 (2014)

59. Besset, T., Poisson, T. \& Pannecoucke, X. Recent progress in direct introduction of fluorinated groups on alkenes and alkynes by means of C-H bond functionalization. Chem. Eur. J. 20, 16830-16845 (2014).

60. Merino, E. \& Nevado, C. Addition of $\mathrm{CF}_{3}$ across unsaturated moieties: a powerful functionalization tool. Chem. Soc. Rev. 43, 6598-6608 (2014).

61. Liang, T., Neumann, C. N. \& Ritter, T. Introduction of fluorine and fluorinecontaining functional groups. Angew. Chem. Int. Ed. 52, 8214-8264 (2013).

62. Chatterjee, T., Iqbal, N., You, Y. \& Cho, E. J. Controlled fluoroalkylation reactions by visible-light photoredox catalysis. Acc. Chem. Res. 49, 2284-2294 (2016).

63. Weix, D. J. Methods and mechanisms for cross-electrophile coupling of Csp ${ }^{2}$ Halides with alkyl electrophiles. Acc. Chem. Res. 48, 1767-1775 (2015).

64. Knappke Christiane, E. I. et al. Reductive cross-coupling reactions between two electrophiles. Chem. Eur. J. 20, 6828-6842 (2014).

65. Everson, D. A. \& Weix, D. J. Cross-electrophile coupling: principles of reactivity and selectivity. J. Org. Chem. 79, 4793-4798 (2014).

66. Li, Z. L., Li, X. H., Wang, N., Yang, N. Y. \& Liu, X. Y. Radical-mediated 1,2formyl/carbonyl functionalization of alkenes and application to the construction of medium-sized rings. Angew. Chem. Int. Ed. 55, 15100-15104 (2016).

67. Liu, Z. et al. Copper-catalyzed acyltrifluoromethylation of alkenes: rapid access to trifluoroethyl indanones and related compounds. Chem. Commun. 53, 6440-6443 (2017).

68. Zultanski, S. L. \& Fu, G. C. Catalytic asymmetric $\gamma$-alkylation of carbonyl compounds via stereoconvergent suzuki cross-couplings. J. Am. Chem. Soc. 133, 15362-15364 (2011)

69. Saito, B. \& Fu, G. C. Enantioselective alkyl-alkyl suzuki cross-couplings of unactivated homobenzylic halides. J. Am. Chem. Soc. 130, 6694-6695 (2008).

70. Fahey, D. R. \& Mahan, J. E. Oxidative additions of aryl, vinyl, and acyl halides to triethylphosphinenickel(0) complexes. J. Am. Chem. Soc. 99, 2501-2508 (1977).

71. Durandetti, M., Devaud, M. \& Perichon, J. Investigation of the reductive coupling of aryl halides and/or ethylchloroacetate electrocatalyzed by the precursor $\mathrm{NiX}_{2}$ (bpy) with $\mathrm{X}-=\mathrm{Cl}-, \mathrm{Br}$ - or $\mathrm{MeSO}_{3}$ and bpy $=2,2^{2}$-dipyridyl New J. Chem. 20, 659-667 (1996).

72. Gutierrez, O., Tellis, J. C., Primer, D. N., Molander, G. A. \& Kozlowski, M. C. Nickel-catalyzed cross-coupling of photoredox-generated radicals: uncovering a general manifold for stereoconvergence in nickel-catalyzed cross-couplings. J. Am. Chem. Soc. 137, 4896-4899 (2015). 


\section{Acknowledgements}

We thank the National Natural Science Foundation of China (21702029), and the "Thousand Plan" Youth program, the Shanghai Sailing Program (17YF1400100), and the Fundamental Research Funds for the Central Universities for financial support.

\section{Author contributions}

X.Z. and H.Y.T. contributed equally to this work. L.C. conceived and designed the project. L.C., X.Z., H.Y.T., L.G., S.Z., and F.L.Q. designed the experiments. X.Z., H.Y.T., L.G., and S.Z. performed the experiments and analyzed the data. L.C. prepared the manuscript.

\section{Additional information}

Supplementary Information accompanies this paper at https://doi.org/10.1038/s41467018-05951-6.

Competing interests: The authors declare no competing interests.

Reprints and permission information is available online at http://npg.nature.com/ reprintsandpermissions/
Publisher's note: Springer Nature remains neutral with regard to jurisdictional claims in published maps and institutional affiliations.

(c) (i) Open Access This article is licensed under a Creative Commons Attribution 4.0 International License, which permits use, sharing, adaptation, distribution and reproduction in any medium or format, as long as you give appropriate credit to the original author(s) and the source, provide a link to the Creative Commons license, and indicate if changes were made. The images or other third party material in this article are included in the article's Creative Commons license, unless indicated otherwise in a credit line to the material. If material is not included in the article's Creative Commons license and your intended use is not permitted by statutory regulation or exceeds the permitted use, you will need to obtain permission directly from the copyright holder. To view a copy of this license, visit http://creativecommons.org/ licenses/by/4.0/.

(C) The Author(s) 2018 